Meta

Journal des traducteurs

Translators' Journal

\title{
Opacité et transparence dans les technolectes bilingues (français-arabe)
}

\section{Leila Messaoudi}

Volume 45, numéro 3, septembre 2000

La traduction dans le monde arabe

URI : https://id.erudit.org/iderudit/003641ar

DOI : https://doi.org/10.7202/003641ar

Aller au sommaire du numéro

Éditeur(s)

Les Presses de l'Université de Montréal

ISSN

0026-0452 (imprimé)

1492-1421 (numérique)

Découvrir la revue

Citer cet article

Messaoudi, L. (2000). Opacité et transparence dans les technolectes bilingues (français-arabe). Meta, 45(3), 424-436. https://doi.org/10.7202/003641ar
Résumé de l'article

L'opacité des terminologies ne s'explique pas par des insuffisances inhérentes aux LS ouLC; elle proviendrait des pratiques traduisantes qui ont tendance à ne retenir que l'aspect générique des technolectes traduits, comme l'illustrent les échantillons empruntés aux domaines agricole et médical, donnant lieu à des confusions fâcheuses.Seule "une traduction conceptuelle (de "concept") [...] assurerait la "transparence " requise pour les technolectes ". "L'évaluation du degré de transparence du technolecte dans la LC consisterait à la fois à mesurer le degré d'appropriation du concept via la LS mais aussi le taux de stratégies d'adéquation mises en œuvre par l'exploitation de moyens propres dont toute langue dispose". 


\title{
Opacité et transparence dans les technolectes bilingues (français-arabe)
}

\author{
LEILA MESSAOUDI \\ Faculté des lettres et des Sciences humaines, Kénitra, Maroc
}

\begin{abstract}
RÉSUMÉ
L'opacité des terminologies ne s'explique pas par des insuffisances inhérentes aux LS ou LC; elle proviendrait des pratiques traduisantes qui ont tendance à ne retenir que l'aspect générique des technolectes traduits, comme l'illustrent les échantillons empruntés aux domaines agricole et médical, donnant lieu à des confusions fâcheuses.

Seule «une traduction conceptuelle (de «concept») [...] assurerait la «transparence» requise pour les technolectes». «L'évaluation du degré de transparence du technolecte dans la LC consisterait à la fois à mesurer le degré d'appropriation du concept via la LS mais aussi le taux de stratégies d'adéquation mises en œuvre par l'exploitation de moyens propres dont toute langue dispose. »
\end{abstract}

\begin{abstract}
Opacity in terminologies is not due to deficiencies inherent in the target language or the source language; it would stem from the translating practices that tend to retain only the generic aspect of the translated technolects, as illustrated by samples borrowed from agricultural and medical fields, bringing about unfortunate confusion.

Only "a conceptual translation (from "concept") [...] would guarantee the "transparency" required for technolects." "The evaluation of the degree of transparency in the target language would consist in measuring both the degree of appropriateness of the concept via the source language, and the percentage of strategy appropriateness used in the implementation of the means particular to each language."
\end{abstract}

\section{MOTS-CLÉS/KEYWORDS}

transparence, opacité, technolecte, terminologie, concept

Dans un précédent travail (Messaoudi 1998), l'étude formelle a montré que la langue cible use de tous les moyens dont elle dispose pour une mise à niveau des technolectes. Deux stratégies sont mises en œuvre. L'une, de compensation, consiste à vouloir combler les lacunes par le recours à l'emprunt de la langue source qui "compense» ainsi un vide terminologique constaté dans la langue cible. L'autre, d'adéquation par laquelle la langue cible utilise son propre système et est en conformité (en adéquation) avec ses règles. On peut noter, à cet effet, pour la langue arabe, une large exploitation morphologique des schèmes intra-radicaux et extra-radicaux ainsi qu'un usage assez fréquent des procédés syntaxiques par la détermination et la juxtaposition. Parfois, la mixonymie est utilisée.

Comme toute langue soucieuse de se développer et d'être en mesure d'assimiler le progrès, la langue arabe use de ces deux stratégies avec une large prédilection pour la seconde en ce qui concerne les procédés formels. Il serait intéressant de poursuivre la recherche au sujet des procédés non formels, c'est-à-dire sémantiques, auxquels la LC a recours pour rendre le «concept» via la LS. 


\section{Sur les procédés non formels}

L'opération traduisante ne peut se contenter d'avoir recours aux seuls procédés formels et de faire l'économie du sens. Le sens est le vecteur déterminant de ce transfert de langue à langue. Il convient donc de s'interroger sur les aspects sémantiques, autrement dit sur les procédés non formels. L'appellation "procédés non formels", appliquée à des aspects sémantiques et l'utilisation même du terme de "procédé» méritent une discussion que nous ne soulevons pas ici. Nous avons déjà eu l'occasion de la faire dans notre thèse de doctorat (Messaoudi 1990). Nous n'y reviendrons pas. Toutefois certaines précautions s'imposent:

- Tout d'abord, la sémantique connaît actuellement des évolutions très intéressantes (voir, par exemple, les travaux de G. Kleiber). Cependant, nous n'en tiendrons pas compte sachant que nous ne pourrions naviguer, avec aisance, dans les méandres théoriques de ce domaine qui n'est point notre champ de spécialité.

- La sociolinguistique et la socioterminologie seraient d'un grand apport et aideraient à saisir certains effets de sens de la LS et leur traitement, par admission, omission ou réinterprétation, par la LC: par exemple, le fait de rendre «verre à madère» de LS par «verre à thé» dans la LC, pour les proportions mentionnées dans les notices pharmaceutiques. Ces paramètres seront sciemment évacués même s'ils constituent des pistes de recherche prometteuses. Les données sur lesquelles nous opérerons sont pour ainsi dire «momifiées» et mises «hors circuit» social et hors acte de communication pour être décortiquées in vitro.

Ces réserves étant faites, nous nous contenterons de distinguer deux sortes de relations sémantiques: les relations intra-signes et les relations inter-signes. Les deux réseaux de sens étant foncièrement différents, ils requièrent deux types de démarches: l'une opère à l'intérieur — ou plutôt dans les limites — de l'unité technolectale, en visant sa décomposition en unités minimales de signification, et ce, à l'instar de la décomposition des segments phonologiques en traits minima; l'autre essaie de déterminer les types de rapports qui relient les unités entre elles (rapports de synonymie, de polysémie, d'homonymie, etc.).

Pour ce qui est de la première démarche, elle est appelée communément «analyse componentielle» dans la tradition anglaise et américaine (Lyons 1978: 257-271, etc.) et «analyse sémique» dans la tradition française (Greimas 1966; Pottier 1963, 1964, 1987, etc.). Il semblerait que la distinction entre les deux appellations provienne du fait que, dans l'analyse componentielle, il soit question d'une configuration sous la forme d'un schéma ou d'un diagramme (Nida 1975) ou d'une toile d'araignée (Lyons 1983). Il s'agirait d'une même approche visant à dégager les traits de sens pertinents et permettant de cerner les différences et les points communs entre les unités examinées. C'est du moins la définition que nous retiendrons. Nous ne prendrons pas part au débat sur les avantages et/ou inconvénients de l'analyse en traits. Les polémiques autour de la validité des traits de sens, leur identification, leur relation au social, à l'acte d'émission et de réception seront délibérément ignorées. En revanche, nous porterons une attention particulière au transfert du sens via l'analyse en traits, lors de leur passage de la LS à la LC. Nous aurons recours au double concept d'opacité et de transparence pour évaluer le degré d'appropriation des contenus conceptuels des technolectes par LC. 
Nous poserons que:

- Dans le processus de traduction, la langue source (LS) est émettrice et la langue cible (LC) réceptrice.

- On parlera de transparence lorsque la LS s'efface et que la LC assume totalement l'unité technolectale.

- On parlera d'opacité lorsque la LS ne s'efface que partiellement et subsiste à travers l'unité technolectale dans la LC.

Comment mesurer la présence de la LS ou son effacement?

- La présence totale de la LS se manifeste par l'emprunt non intégré par LC (p. ex. /laktumitr/, /hydrumitr/), autrement dit lorsque la LC a recours à des stratégies de compensation.

- La présence partielle de la LS se manifeste par le calque du signe linguistique (traduction littérale).

- L'effacement total de la LS se manifeste par le type de lien construit par la LC avec le référent objet (concept) : traduction conceptuelle.

Par ailleurs, l'hypothèse fondamentale concernant le signe technolectal est qu'il est univoque et montre une prédilection certaine pour l'aspect référentiel (renvoie à l'objet dans la réalité). Or, cet aspect référentiel, dans le cas de la traduction, n'est jamais saisi directement. Le détour obligé par la LS pourrait en fausser l'interprétation si l'approche du sens ne se fait pas de façon appropriée.

Afin de vérifier cette hypothèse, nous allons traiter en pratique quelques exemples puisés dans les corpus de technolectes bilingues sur lesquels nous avons eu l'occasion de travailler. Nous retiendrons deux échantillons: l'un tiré du domaine agricole (AGR); l'autre, du domaine de la santé (SAN).

\section{De quelques exemples}

Nous avions constitué un corpus à partir de sources lexicographiques bilingues (Messaoudi 1990: 188-222) en répertoriant les noms d'outils et de machines dans le domaine agricole. Pour les besoins de l'analyse, nous en avons extrait un échantillon relatif aux «instruments qui servent à couper».

Pour construire cet échantillon, nous nous sommes posé les questions suivantes:

- Faut-il employer le terme «instrument» avec un sens large, englobant à la fois les outils manuels et les machines?

- Faut-il retenir tout instrument présentant matériellement, dans le référent-objet, qui peut être illustré par un dessin, un équipement pour couper (p. ex., une lame)?

- Faut-il prendre en considération la seule fonction de «couper» ou bien faut-il y intégrer le résultat obtenu? Par exemple, ce qui est coupé, est-il jeté ou conservé? De ce point de vue, l'opération de moissonner pourrait renvoyer à " couper + conserver»; en revanche, l'opération d'émonder, à " couper + jeter ». Cette distinction sera-t-elle pertinente pour cerner la fonction de l'outil? C'est ce qui différencierait, par exemple, une «moissonneuse» d'un «émondoir».

- La fonction de couper englobe-t-elle celle d'arracher?

- Faut-il prendre en considération les gestes, la position, etc. de l'agent humain qui manipule l'instrument sachant que l'objet technique définit, par sa configuration, le rôle attribué à l'acteur et le mode de relation avec le «réel»? (Leroi-Gourhan 1964; Akrich 1987). 
Pour répondre à ces questions, nous avons opté pour les choix suivants :

- La distinction entre outils manuels et machines sera retenue.

- Les traits descriptifs formels des instruments (à manche long ou court, à lame plate ou courbe, à disques rotatifs, etc.) qui servent à les identifier seront pris en considération. Ces traits rappellent les descripteurs des prospectus de vente des objets manufacturés ou encore l'étiquetage des objets après les fouilles archéologiques.

- La fonction "couper» est à dissocier du but vers lequel est orientée cette action. La distinction entre les buts de «couper» ne sera donc pas maintenue.

- La fonction de couper n'englobera pas celle d'arracher.

- Le mode de relation à l'agent humain ne sera pas considéré même si, ailleurs, il peut être mentionné dans la définition lexicographique.

Ces précisions étant faites, nous essaierons de dégager des réseaux relationnels dans le micro-champ que nous avons examiné et qui est tiré de sources lexicographiques bilingues (français-arabe) ou trilingues (français-anglais-arabe) (Messaoudi 1990). Nous savons combien est décriée la définition des dictionnaires (Pottier 1964). Pourtant, elle constitue une référence incontournable (Chaurand et Mazière 1990). Il est vrai que le dépouillement lexicographique peut être complété par d'autres sources documentaires comme le prospectus sur les caractéristiques et les modes d'utilisation des objets (outils ou machines), tout comme il doit être confronté au savoir et savoir faire des spécialistes (les ingénieurs et techniciens agronomes) et des utilisateurs (les paysans). Toutefois, dès lors que l'on essaie d'intégrer le «terrain», la diglossie arabe, en marche depuis des siècles, réoriente l'approche et l'on ne peut considérer le seul niveau standard de l'arabe. Il faudrait prendre en compte l'arabe dialectal local pour que l'étude soit globale. Nous ne l'avons pas fait ici puisque nous avons tenu à étudier un corpus écrit et bilingue. L'approche globale (écrit + oral) nous aurait entraîné vers un autre type d'étude. Ce travail sur le terrain est en cours (un technolecte agricole en arabe dialectal marocain dans le parler des Jbala (Maroc du Nord-Ouest) [à paraître]) et des comparaisons ultérieures pourront être faites.

Après cette digression, nécessaire pour cerner davantage le micro-champ envisagé, nous présentons l'exemple d'application (AGR).

\section{L'exemple AGR et l'analyse en traits}

Nous poserons que les instruments qui servent à couper dans le domaine agricole se subdivisent en deux: les outils et machines. Les traits descripteurs des premiers sont: manche long ou court, lame plate ou courbe. Pour les secondes, est vue comme pertinente la distinction entre les machines à une fonction de celles qui en cumulent plusieurs.

On peut remarquer que les traits attribués aux outils sont dégagés de la forme de ces derniers. Tandis que les traits des machines sont tirés du nombre de fonctions qu'elles sont appelées à assurer. Le tableau suivant récapitule les traits retenus :

Instruments pour couper

\begin{tabular}{|l|l|l|l|l|l|}
\hline \multicolumn{2}{|l|}{ Outils } & \multicolumn{2}{l|}{ Machines } \\
\hline \multicolumn{2}{|l|}{ Manche } & \multicolumn{2}{l|}{ Lame } & Unifonctionnelle & Plurifonctionnelle \\
\hline Long & Court & Courbe & Plate & & \\
\hline
\end{tabular}


Nous commencerons par les noms d'outils (manuels), en prenant l'exemple suivant:

\begin{tabular}{|l|c|c|c|c|}
\hline Nom d'outil & Manche long & Manche court & Lame courbe & Lame plate \\
\hline $\begin{array}{l}\text { Ar: /minjal/ } \\
\text { Fr: faucille }\end{array}$ & - & + & + & - \\
\hline $\begin{array}{l}\text { Ar: } / \text { miqdab/ } \\
\text { /mihsàd/ } \\
\text { Fr: faux }\end{array}$ & + & - & + & - \\
\hline $\begin{array}{l}\text { Ar: } / \text { miqdabah/ } \\
\text { Fr: fauchon }\end{array}$ & + & - & + & - \\
\hline
\end{tabular}

Note: Dans le tableau, l'abréviation Ar renvoie à arabe (standard) et Fr à français.

Remarques :

- Les deux appellations /miqdab/ et /mihsàd/ sont des variantes.

- Dans la grille, les deux instruments «faux» et «fauchon» sont décrits par les mêmes traits. Pourtant il ne s'agit pas des mêmes outils. Ceci montre que les traits retenus ne sont guère suffisants pour rendre compte des différences entre les outils, pourtant distincts. Le "fauchon» est une "faux armée d'un râteau». Faudrait-il ajouter un trait supplémentaire, une nouvelle case à la grille, du genre: armé ou non armé?

- Les traits que nous avons testés ne fonctionnent que pour un type donné d'instruments et n'englobent pas, par exemple, le «sécateur» ou la «cisaille».

Un remaniement des traits descripteurs est donc nécessaire. Une formulation adéquate est requise. Peut-être que l'opposition "générique» versus «spécifique», comme l'appliquent les terminologues notamment (Dubuc 1980), pourrait coiffer l'ensemble des traits et aider à décrire le maximum d'outils. Sur quels critères se fonder pour décréter que tel ou tel trait est générique et tel autre spécifique?

Nous nous référerons à Pottier (1974 et 1987). Pour la cohérence terminologique, nous restituons les contextes dans lesquels il utilise ces deux concepts. Il s'agit de l'analyse sémantique en traits. Ses postulats sont les suivants:

- «La substance du signifié d'un signe (sé) est constituée par un ensemble de traits distinctifs de signification. Au niveau du signe minimal, ou morphème, l'ensemble est appelé le sémème et chaque trait est un sème:

Sémème $=$ sème 1 , sème $2, \ldots$ sème $n$. (Pottier 1974: 29)

- «Les sèmes ne sont pas tous de même nature. Les uns sont dénotatifs et déterminent d'une façon stable et avec une vaste assise sociale la signification d'un signe (ex.: avec moteur pour autobus). D'autres sont connotatifs et caractérisent de façon instable et souvent individuelle la signification d'un signe (être secoué pour autobus).»(Pottier 1974: 29-30)

Il est intéressant de relever, à ce stade, que l'opposition entre dénotation et connotation repose sur une double dichotomie:

- $\quad$ stabilité versus instabilité

- $\quad$ vaste assise sociale versus individuel.

Ces aspects, pour être vérifiés, devront reposer sur des enquêtes auprès des usagers sur la réception et la production sémantique (Melka Teichrow 1989). Nous 
avons déjà signalé que nous n'allions pas tenir compte de ces paramètres en restreignant volontairement le champ d'analyse.

Pour en revenir au concept bipolaire de générique versus spécifique, on constatera qu'il est relié par Pottier à celui de dénotation: "Les sèmes dénotatifs sont soit spécifiques, lorsqu'ils permettent de distinguer deux sémèmes voisins (ex.: "deux» dans biréacteur en face de triréacteur); soit génériques lorsqu'ils indiquent l'appartenance à une catégorie générale (ex. : «matériel» pour biréacteur...).» (Pottier 1974: 30).

Les sèmes génériques et spécifiques sont tous deux reliés aux sèmes dénotatifs qui sont, théoriquement, les seuls qui importent dans l'approche des unités technolectales. Nous avons déjà énoncé, à la suite de Guilbert (Messaoudi 1990), les traits définitoires du signe technolectal, parmi lesquels celui de l'univocité du signe. À l'inverse de la communication poétique, qui requiert les sèmes connotatifs, la communication technolectale exigerait, quant à elle, uniquement les sèmes dénotatifs.

Pour en revenir à la grille des outils, nous pourrions la remanier en posant que le sème générique est leur appartenance à une catégorie générale dont la fonction est de couper. Les sèmes spécifiques seront plus détaillés afin d'englober un plus grand nombre d'outils. Les sèmes complémentaires sont armé versus non armé et à une lame versus à deux lames. La nouvelle grille se présente comme suit:

\begin{tabular}{|l|c|c|c|c|c|c|c|}
\hline Outils & $\begin{array}{c}\text { Manche } \\
\text { long }\end{array}$ & $\begin{array}{c}\text { Manche } \\
\text { court }\end{array}$ & $\begin{array}{c}\text { Une lame } \\
\text { courbe }\end{array}$ & $\begin{array}{c}\text { Une lame } \\
\text { plate }\end{array}$ & $\begin{array}{c}\text { Deux lames } \\
\text { courbes }\end{array}$ & $\begin{array}{c}\text { Deux lames } \\
\text { plates }\end{array}$ & Armé \\
\hline $\begin{array}{l}\text { Ar: } / \text { minjal/ } \\
\text { Fr: faucille }\end{array}$ & - & + & + & - & - & - & - \\
\hline $\begin{array}{l}\text { Ar: /miqdab/ } \\
\text { ou /mihsad/ } \\
\text { Fr: faux }\end{array}$ & + & - & + & - & - & - & - \\
\hline $\begin{array}{l}\text { Ar: /miqdabah/ } \\
\text { Fr: fauchon }\end{array}$ & + & - & + & - & - & - & + \\
\hline $\begin{array}{l}\text { Ar: /miqrad/ } \\
\text { Fr: cisaille }\end{array}$ & - & - & - & - & - & + & - \\
\hline $\begin{array}{l}\text { Ar: /miqassu } \\
\text { lhadà iq/ } \\
\text { Fr: sécateur }\end{array}$ & - & - & - & - & + & - & - \\
\hline
\end{tabular}

Quels que soient les reproches adressés à l'analyse en traits, elle s'avère utile et efficace parce qu'elle conduit à des distinctions utiles, fondées sur les sèmes communs et les sèmes différentiels. Il va de soi que la grille devra s'adapter à chaque analyse et sera dégagée a posteriori des données examinées. Il n'est nullement question de transposer telle ou telle grille mais bien de la construire et de la déconstruire pour la remanier autant de fois que de besoin. La grille présentée ci-dessus rend bien compte, à notre avis, des distinctions à opérer entre les dénominations et, de ce fait, permet de déceler les différents réseaux sémantiques qui se tissent entre les termes. 
En passant en revue les termes retenus dans la grille, nous nous apercevons que /minjal/ fonctionne dans d'autres ensembles. Il désigne l'objet "faucille», qui sert à couper l'herbe et les céréales, mais aussi la «serpe », qui sert à couper les branches et la «sape», variante du «faux». De même, /miqdab/, qui désigne l'objet «faux», se trouve employé pour «serpe» et pour «émondoir». /mihsad/, variante de /miqdab/, qui désigne l'objet «faux», sert quant à lui à la dénomination de l'objet «moissonneuse».

S’il est vrai que tous ces outils sont voisins dans la mesure où ils appartiennent au même domaine (l'agriculture) et ont été regroupés selon le sème générique : «instruments qui servent à couper», il n'en demeure pas moins que la confusion règne puisque l'on ne distingue plus entre «la faucille», «la serpe», la «sape» et le «faux» lorsqu'on utilise /minjal/; de même, on n'opère plus de différence entre «le faux», «la serpe», «l'émondoir» et «la moissonneuse » lorsqu'on utilise le terme /miqdab/.

Ainsi, sur le plan terminologique, en ce qui concerne ces unités dans le technolecte arabe, tout se passe comme si seul le sème générique était pertinent tandis que les sèmes spécifiques ont été relégués à l'oubli. Et l'on peut multiplier les exemples de ce type.

\section{Polysémie non fonctionnelle et homonymie}

Et il en découle une polysémie non fonctionnelle comme source de perturbations, tout particulièrement lorsqu'il s'agit du même domaine. Et, de toute évidence, si la polysémie est synonyme d'économie dans le lexique «ordinaire» et si, effectivement, comme le signale Picoche (1986: 4), «la polysémie suppose un mécanisme sémantique extrêmement puissant qui rend un seul et même signe capable de balayer une partie importante de l'expérience humaine », il n'en va pas de même dans le technolecte où, au contraire, la polysémie, au lieu d'être une source de richesse lexicale peut devenir une source d'erreur. Mieux vaut l'éviter si l'on veut assurer la transparence du technolecte. Il serait préférable d'opter pour une relation d'homonymie dans les cas où l'on a affaire à un même signifiant employé dans deux ou plusieurs domaines différents, comme dans l'exemple de l'utilisation de "faux» en agriculture et en médecine, même si l'analyse en traits révèle quelques sèmes communs. Le «faux» est une dénomination de type métaphorique, qui renvoie à ce que nous avons appelé «mode de désignation symbolique» fondé sur une relation «substitutive» exprimée par «comme» (Messaoudi 1995) dans la mesure où la référence est faite à la forme de l'outil pour désigner dans le cerveau «la partie de la dure-mère, formant une cloison médiane entre deux hémisphères » (Garnier et Delamare 1989).

$\mathrm{Au}$ reste, l'analyse en traits n'est pas la seule méthode pour distinguer polysémie et homonymie. Le test de la commutation et celui de l'association avec des éléments identiques ou non identiques fourniront des critères utiles pour les différencier. À cet effet, André Martinet (1974: 44) a noté: «Si deux segments significatifs s'opposent dans un environnement contextuel et situationnel identique, il s'agit, sans qu'il puisse y avoir le moindre doute, de deux monèmes différents. Mais, s'il s'agit de segments apparaissant toujours dans des environnements différents, la preuve de leur identité ou leur non-identité ne pourra être fournie que par l'identité ou la nonidentité de leurs rapports avec les autres unités de leur système respectif, c'est-à-dire celles susceptibles de commuter avec chacun d'eux.» 
Nous avons souligné le terme «situationnel» dans la citation ci-dessus vu son importance pour les technolectes. En effet, dans le cas de l'homonymie, les éléments situationnels apportent un éclairage précieux et permettent de désambiguïser. Parmi ces éléments, celui du «domaine» est assez déterminant. Nous avons donné l'exemple de «faux» dans deux domaines différents : l'agriculture et la médecine. Si le linguiste interprète ce cas comme étant une polysémie en cherchant des sèmes communs aux deux termes, pour le terminologue et l'usager, le sens technolectal primera et les deux termes seront vus comme homonymes même si, sur le plan diachronique, la racine étymologique est «identique». D'ailleurs, le recours à l'étymologie n'est pas apprécié de la même façon par les linguistes. Pour les uns, il est préférable de ne pas en tenir compte. Par exemple, Martinet (1974:40) a relevé à ce sujet: "Il nous faut [...] résolument bannir toute utilisation de l'étymologie dans un examen fonctionnel du problème de l'homonymie et de la polysémie.»

À l'opposé, pour les autres, le recours à l'étymologie est le seul moyen pertinent pour distinguer homonymes et polysèmes. Ainsi, Jean et Claude Dubois (1971: 37) soulignent: "La distinction qu'on institue entre les homonymes et les polysèmes n'a de valeur que dans une explication de type diachronique. Sur le plan du fonctionnement de la langue, cette distinction perd de sa pertinence. »

Toutefois, si cette distinction perd de son importance sur le plan purement synchronique, il convient, à notre avis, de la maintenir dans le champ des technolectes. Seront ainsi interprétés comme homonymes les termes qui ont un même signifiant mais qui appartiennent à des domaines différents. En ce sens, le terme «faux» ne sera pas considéré comme polysémique. Nous aurions affaire à «faux 1 » dont le domaine d'emploi sera Agriculture (AGR) et «faux 2 » dont le domaine d'emploi sera Médecine (SAN). Cette nécessité de tenir compte des domaines d'emploi n'échappe plus aux terminologues qui l'intègrent comme paramètre parmi les traits définitoires du technolecte. Cet aspect n'a pas non plus échappé aux lexicographes qui n'ont pas manqué de le mentionner. La preuve en est la liste des domaines d'emploi (DE) présente dans les dictionnaires de langue aussi bien que - et de plus - dans les dictionnaires spécialisés.

La question que nous pourrions nous poser est de savoir comment réagit la LC face à une homonymie dans la LS?

Pour le cas de "faux», la LC a procédé à un calque par traduction littérale. Le mot utilisé dans les deux occurrences AGR et SAN est /minjal/. Il se trouve ainsi proposé comme l'équivalent de:

- «faux» des deux domaines (AGR) et (SAN),

- «faucille» (AGR),

- «sape» (AGR).

Il se trouve que la traduction littérale peut être heureuse parfois! Le calque dans le cas de "faux» s'explique par le fait que le référent renvoie à la forme de "faux». Mais les difficultés surgissent en arabe lorsqu'on constate que /minjal/ renvoie aussi bien à «faux» qu'à «faucille» qu'à «serpe» et qu'à «sape». Ces outils ont-ils la même forme dans la réalité? Un agriculteur les confondra-t-il ? La réponse est non. Nous sommes face à une interprétation de la LC fondée sur une synonymie entre faux, faucille, sape et serpe. Fausse synonymie, introduite par une traduction qui ne se fonde que sur le sème générique. Tous ces objets ont pour fonction commune de 
couper les céréales, mais ils présentent des caractéristiques qui les distinguent comme la longueur du manche, l'aspect courbe ou non de la lame, etc.

\section{L'exemple de SAN et la fausse synonymie}

Rappelons que, sur le plan théorique, une synonymie parfaite qui s'appuierait sur une relation d'identité des traits de sens de deux ou plusieurs unités lexicales est très rare. Cette observation a été émise par plus d'un chercheur (Schogt 1976). Patry et Ménard (1990: 34) notent: «Des mots en relation de synonymie n’ont pas nécessairement le même sens lexical pour toutes les acceptions qu'on leur reconnaît».

D'ailleurs, ces deux auteurs citent les distinctions opérées par D.A. Cruse (1986) entre absolute synonymy, cognitive synonymy et plesionymy, qu'ils traduisent respectivement par synonymie complète, synonymie de dénotation et fausse synonymie.

La synonymie complète, rarissime, exigerait un comportement comparable en tous points de la chaîne et les mêmes types d'appréciation de la part des locuteurs quant au contenu affectif, émotif, etc. Il est évident que ce sont là des conditions de production discursive mais, comme nous l'avons énoncé plus haut, nous ne tiendrons pas compte du fonctionnement des unités technolectales dans le discours pour des raisons méthodologiques mais aussi en raison du statut sociolinguistique particulier de l'arabe standard qui — comme tout standard — est la langue de tous mais qui n'est pas utilisé spontanément dans le discours, d'autant que le discours technolectal fonctionne souvent en bilingue (français-arabe) ou en diglossie (arabe standard-arabe dialectal).

Pour en revenir aux autres relations synonymiques, nous pourrions nous demander de quel type participent celles que nous avons détectées dans la LC dans le corpus SAN recueilli dans les notices pharmaceutiques? Prenons par exemple les différents termes utilisés pour désigner les différentes formes de présentation des médicaments. Nous constaterons que:

- /unbuba/ est employé pour «ampoule» mais aussi pour «tube»;

- /qinnina/ désigne "ampoule», «étui » mais aussi «tube»;

- /qàrura/ renvoie à «flacon» mais aussi à «ampoule».

Les termes «tube», "ampoule», "flacon» se trouvent présentés comme des synonymes; or, ils sont loin de l'être. Il s'agit là d'une fausse synonymie. La synonymie absolue ou dénotative ( $\mathrm{du}$ type «capsule»= "gélule») ne pose pas de problème d'ambiguïté et les risques de confusion sont minimes à partir du moment où la LC a repéré cette synonymie dans la LS. D’ailleurs, "gélule» est rendu en arabe par /kapsula/, emprunté de «capsule». En outre, le recours, en arabe, à une même racine pour les termes appartenant à un même sous-domaine peut être une approche intéressante car de type analogique mais, non maîtrisée, elle peut être source d'ambiguïté. Par exemple, le fait d'employer les dérivés /mulabbasa/ "gélule», "comprimé dragéifié » et /labbusa/ «suppositoire» provenant de la même racine / لlbs/ «notion d'habiller» ne fait que semer la confusion. On peut aisément confondre suppositoire et comprimé lors de la médication! Les schèmes à eux seuls ne suffisent pas toujours à maintenir distincts les termes, car leur valeur sémantique n'est pas toujours stable. Et le locuteur non averti pourra aisément confondre un suppositoire et un comprimé! Si certains schèmes présentent une certaine régularité dans leur fonctionne- 
ment sémantique - comme les schèmes désignant les noms d'outils ou de machines -, il n'en va pas de même pour tous les autres.

En revanche, ce qui crée des perturbations, c'est la fausse synonymie. Si cette dernière ne porte pas à conséquence dans le langage "ordinaire», dans la communication technolectale, elle peut provoquer de graves préjudices, par exemple de considérer comme des synonymes des noms de maladie: la diphtérie, l'amygdalite et l'angine, traduites par /iltihabu llawazatayn/ ou encore d'employer /qawba'/ aussi bien pour l'eczéma que pour l'herpès. Pourtant, ces maladies sont distinctes.

À titre d'exemple, on peut se reporter à la définition d' « eczéma » et d' " herpès»:

- l'eczéma est une «lésion cutanée caractérisée par un placard rouge vif, légèrement surélevé, sur lequel apparaissent rapidement des groupes de petites vésicules transparentes [...]; il est ordinairement l'effet d'une irritation interne ou externe» (Garnier et Delamare 1989).

- l'herpès désigne « des lésions cutanées consistant en vésicules transparentes, du volume d'une grosse tête d'épingle, réunies [...] dans un même groupe et entourées d'une auréole rouge. L'herpès est dû à un virus.» (Garnier et Delamare 1989)

Une analyse en traits permettra de dégager le sème générique «catégorie des maladies de la peau à lésions» et comme sèmes spécifiques «mode de manifestation» (vésicules transparentes + taille des vésicules) et "origine» (irritation dans un cas et virus dans l'autre).

Que déduire de cet exemple et d'autres encore? Comment se comporte la LC, en l'occurrence la langue arabe? Le degré de transparence requis est-il atteint? Faut-il le mesurer par rapport à la LS comme on l'a supposé au début de cette étude? Ou bien faut-il essayer de l'évaluer par rapport au «concept» qui n'est autre qu'un «objet référent» de la réalité?

\section{Pour une théorie conceptuelle de la traduction du technolecte}

L'unité technolectale, quelle que soit la langue considérée, entretient une relation étroite avec l'objet-référent de la réalité. Le signe technolectal est toujours motivé par rapport au référent. Une traduction qui délaisserait ce lien mutilerait l'unité technolectale de ce qui fait son essence: «le concept» ou bien «la notion» comme cela est l'usage chez les terminologues qui distinguent d'ailleurs entre divers types de notions (cf. Vocabulaire systématique de terminologie 1985). Le lien entre le signifié et le référent se retrouve implicitement dans le concept. Le signifié n'en est qu'une expression minimale. Nous postulons que le signifié qui appartient à un signe linguistique d'une langue particulière «n'est pas le concept», mais il en représente une partie.

On sait depuis l'enseignement aristotélicien que le concept correspond à la représentation de l'élément commun aux membres d'une même catégorie. Le langage fait largement usage de ces représentations générales pour permettre une communication à moindre coût. Paradoxalement, le général au détriment du particulier, l'indéterminé au détriment du précis augmentent les possibilités d'application et constituent un procédé économique dans la communication ordinaire. Par exemple, «table» s'applique aussi bien à "table de jeux», "table de multiplication », "table d'opérations » qu'à table en bois, en marbre, en métal, à surface ovale, ronde carrée, etc. Il semblerait que, dans le langage ordinaire, nous communiquons le plus souvent par 
représentations générales qui sont précisées la plupart du temps par le contexte linguistique et la situation de communication.

Cassirer (1977: 257) a d'ailleurs souligné que: «[...] le mot oiseau ne correspond plus à aucun contenu intuitif à détermination pleine, il ne retient que certaines esquisses imprécises de la figure, associées à la représentation vague du mouvement de vol, si bien qu'un enfant peut donner le nom d'oiseau au scarabée volant ou au papillon». Il en va cependant tout autrement lorsqu'il s'agit de la communication technolectale: c'est le particulier, le précis et le déterminé qui l'emportent. En réalité, le fait de "conceptualiser», aussi bien dans le langage ordinaire que dans le technolecte, relève de la même opération qui consiste à abstraire le(s) élément(s) commun(s). Toutefois, dans un cas, on se contente d'une esquisse tandis que, dans l'autre, on requiert un tracé à bords précis et à traits spécifiques. On peut se référer, encore une fois, à Cassirer (1977: 257): "Quant au concept mis en ouvre par la science, il connaît le même développement et les mêmes conditions. Sa seule différence par rapport aux concepts naïfs du langage et de l'image commune du monde consiste en ce que la procédure qui, dans cette dernière sphère, opère inconsciemment, s'exerce cette fois en pleine conscience critique.»

Dans le domaine des technolectes et dans l'opération traduisante de ces derniers, il s'agit d'une intervention consciente et volontaire. Le traducteur se devrait d'être d'une vigilance extrême. Il ne s'agit point de prôner le principe du traduttore traditore de la traduction littéraire! La traduction de technolecte exige la prise en compte d'un certain nombre de principes:

1. Traduire le concept et ne pas se contenter de la traduction du signifié.

2. Le concept dans le domaine technolectal est censé revêtir un aspect consensuel (universel pour les concepts théoriques ou régional pour certains outillages ou industries, etc.).

3. Il importe de dégager les traits génériques et spécifiques à partir de l'observation de l'objet-référent. Généralement, ce dernier est [+concret] (par concret, on entend visible, palpable, audible... en un mot, perceptible) et/ou [+matériel]. Les traits descripteurs sont en principe aisément cernables. Les difficultés sont accrues en contexte bilingue. Les traits ne sont pas directement saisis. Leur appréhension ne pourra se faire que par le truchement de la LS.

4. Ne pas se contenter du(des) sème(s) générique(s) et veiller à bien distinguer les unités en s'appuyant sur les sèmes spécifiques.

5. Pour une langue à schèmes comme l'arabe, il faudrait maîtriser l'emploi des schèmes les plus productifs afin de les utiliser de façon adéquate, sinon ils seraient sources de «bruit».

\section{Conclusion}

Ainsi, le problème de la transparence ou de l'opacité n'est pas tant la présence ou non de la LS dans la LC, comme nous l'avions supposé au départ. Parfois, certains calques peuvent se révéler pertinents. C'est plutôt la saisie du concept dans l'opération traduisante qui fait souvent défaut dans ce domaine où sévit la traduction approximative, fondée sur les seuls sèmes génériques, comme nous l'avons vu pour la langue arabe. Ce n'est point la LS qu'il faudrait incriminer pour une quelconque infirmité; ce n'est pas non plus la LC qu'il faut tenir pour coupable, en prétextant sa «faiblesse inhérente» à servir de support à l'expression technolectale. Ce sont les opérateurs 
humains (traducteurs, spécialistes et terminologues) qu'il faudrait mettre en garde contre une traduction, par abstraction, du seul signifié linguistique - duquel, de surcroît, seul l'aspect générique est retenu. Il conviendrait d'attirer l'attention sur la nécessité d'une traduction conceptuelle (de «concept») qui seule assurerait la «transparence» requise pour les technolectes. Si l'abstraction est naturelle par essence au langage, dans le technolecte elle ne peut être que porteuse de perturbations et de confusions. Nous avons vu dans cette étude quelques exemples d'outils pris les uns pour les autres ou des maladies confondues. Une traduction scientifique ne saurait tolérer cela.

Et nous reprendrons, à cet effet, cette observation de Cassirer $(1977: 258):$ « [..] la science $[. .$.$] a pour fonction de supprimer l'équivoque, ce qu'elle fait en sélection-$ nant le matériel perceptif au nom de certaines prescriptions dont elle fixe le contenu dans des définitions ayant une validité universelle».

La langue arabe comme LC est apte à exprimer toute chose comme toute langue. Toutefois, l'effort requis n'est pas réellement fourni en particulier pour les technolectes pour lesquels la traduction des seuls sèmes génériques peut être dangereuse et ne permettra pas une réelle promotion de l'arabe comme support des technolectes et de la science en général. Pourtant, cette langue l'a bien été par le passé, elle a bien servi, via la traduction, de médium des concepts scientifiques!

L'évaluation du degré de transparence du technolecte dans la LC consisterait à la fois à mesurer le degré d'appropriation du concept via la LS mais aussi le taux de stratégies d'adéquation mises en œuvre par l'exploitation de moyens propres dont toute langue dispose. Ceci présuppose plusieurs études appliquées par domaines et sous-domaines, recherches qu'il conviendrait de multiplier pour une réelle évaluation. Mais il importe surtout que les traducteurs prennent conscience véritablement de l'importance de la traduction conceptuelle. C'est à ce prix que la transparence sera assurée!

Il convient de signaler aussi le renouveau des théories sémantiques et en particulier l'apport du cognitivisme qui pourra peut-être contribuer à affiner ces analyses et à améliorer l'opération traduisante en domaine technolectal.

\section{RÉFÉRENCES}

Aкrich, M. (1987): «Comment décrire les objets techniques», Technique et cultures, Paris, La Maison des Sciences de l'Homme.

CAssirer, E. (1977): Substance et fonction. Éléments pour une théorie du concept, Paris, Éditions de Minuit.

Chaurand, J. et F. Mazière, dir. (1990): La définition, Paris, Larousse.

Cruse, D. A. (1986) : Lexical Semantics, Cambridge, Cambridge University Press.

Garnier et Delamare (1989): Dictionnaire des termes de médecine, Paris, Maloine.

Duвois, J. et C. (1971): Introduction à la lexicographie: le dictionnaire, Paris, Larousse.

Duвors, D., dir. (1991): Sémantique et cognition, Paris, CNRS.

Dubuc, R. (1980): Manuel pratique de terminologie, Paris, CILF.

Felber, H. (1984): Terminology manual, Paris, UNESCO.

Germain, C. (1981): La sémantique fonctionnelle, Paris, PUF.

Greimas, A.-J. (1966): Sémantique structurale, Paris, Larousse.

Hagège, C. (1982): "Voies et destins de l'action humaine sur les langues", La réforme des langues, Hambourg, Bruske.

KLeiber, G. (1990): La sémantique du prototype, Paris, PUF. 
Leroi-Gourhan, C. (1964): Le geste et la parole, Paris, Albin Michel.

Lyons, J. (1978): Éléments de sémantique, Paris, Larousse.

- (1981): Language, Meaning and Context, London, Fontana.

Martinet, A. (1974): «Homonymes et polysèmes», La linguistique, fasc. 2.

Melka Teichrow, F. J. (1989): Les notions de réception et de production dans le domaine lexical et sémantique, Berne.

Messaoudi, L. (1986): "L'utilisation de la base de données Lexar (de l'IERA) dans la recherche des emprunts dans la terminologie scientifique arabe», Dialogue entre la langue arabe et la langue française, Paris, CILF.

— (1990): Des technolectes, thèse, Université de Paris V.

— (1995): «Linguistique et traduction. Le cas des technolectes», Traduction et interprétation des textes, Rabat, Publications de la Faculté des Lettres et des Sciences Humaines.

— (1998): "Technolectes bilingues (français-arabe) et modes de dénomination ", La Mémoire des mots (T. Baccouche, S. Mejri et A. Clas, dir.), Revue Tunisienne des Sciences Sociales, 117, Tunis, CERES.

- (à paraître): Un technolecte agricole arabe dialectal marocain dans le parler des Jbala.

NidA, E. (1975): Componential Analysis of Meaning. An Introduction to Semantic Structures, ParisThe Hague, Mouton.

Patry, R. et N. Ménard (1990): «La synonymie de la langue est-elle celle du discours?», La linguistique, fasc. 1.

Picoche, J. (1977): Précis de lexicologie française, Paris, Nathan. 